\title{
Heterogeneidade Cognitiva nas Dificuldades de Aprendizagem da Matemática: Uma Revisáo Bibliográfica
}

\author{
Cognitive Heterogeneity in Mathematical Learning Difficulties: A Literature Review
}

Vitor Geraldi Haase ${ }^{\mathrm{I}}$

Annelise Júlio Costa ${ }^{\mathrm{I}}$

Andressa Moreira Antunes ${ }^{\mathrm{I}}$

Isabella Starling Alves ${ }^{\mathrm{I}}$

\section{Resumo}

O progresso tecnológico e econômico de um país associa-se a melhorias na qualidade de vida à custa de uma demanda crescente do desempenho cognitivo e acadêmico da populaçáo. As dificuldades de aprendizagem da matemática (DAM) representam um risco, diminuindo potencialmente o capital cognitivo de uma sociedade. Os diagnósticos e intervenções neuropsicológicos melhoram potencialmente o funcionamento cognitivo e o bem-estar dos indivíduos. Pesquisas são necessárias para um planejamento efetivo e equitativo de políticas públicas. Os critérios diagnósticos atuais das dificuldades de aprendizagem são estatísticos e arbitrários, resultando em uma heterogeneidade clínica e em um alto índice de comorbidades. Neste artigo, foram analisados, através de uma revisão de literatura, os possíveis mecanismos cognitivos envolvidos nas DAM e em outros distúrbios relacionados. Conclui-se que o processamento fonológico, a memória de trabalho, o processamento visoespacial e o senso numérico são domínios potencialmente subjacentes às DAM. Mais estudos longitudinais e integrativos que avaliem simultaneamente a contribuição destes fatores são necessários.

Palavras-chave: Dificuldades de aprendizagem da matemática; senso numérico; memória de trabalho; habilidades visoespaciais; revisão.

\section{Abstract}

Technological and economic progress is associated to higher living standards at the expense of growing demands on cognitive/academic performance. Mathematical learning difficulties (MLD) represent a risk factor, potentially diminishing a society's cognitive capital. Neuropsychological diagnosis and interventions potentially improve cognitive functioning and well-being. Research data are required to plan effective and equitable public policies. Current diagnostic criteria for learning difficulties are statistical and arbitrary, resulting in clinical heterogeneity and high comorbidity rates. In this paper, we discuss possible cognitive mechanisms involved in MLD and their relationship to other disorders through a literature review. It is concluded that phonological processing, working memory, visuospatial processing and number sense are domains potentially underlying MLD. More longitudinal and integrative studies, simultaneously assessing the contribution of these several factors are required.

Keywords: Mathematical learning disabilities; number sense; working memory; visuospatial abilities; review.

${ }^{\mathrm{I}}$ Universidade Federal de Minas Gerais

As transformaçóes tecnológicas e econômicas se associam a melhorias na qualidade de vida (FelderPuig, Baumgartner, Topf, Gadner \& Formann, 2008), às custas, porém, de uma demanda crescente por desempenho cognitivo. Uma comissáo do parlamento britânico recentemente elaborou o conceito de capital mental (Cooper, Field, Goswami, Jenkins \& Sahakian, 2010), o qual diz respeito às consequências econômicas da qualificação intelectual da população. As dificuldades de aprendizagem escolar constituem um dos principais agravos ao capital mental de uma comunidade.

Se as dificuldades de aprendizagem escolar constituem um agravo em potencial para o capital mental de uma sociedade e, ao mesmo tempo, uma oportunidade de atuar preventivamente, há necessidade de dados de pesquisa que fundamentem as políticas públicas na área de educação e saúde. A neuropsicologia dos transtornos de aprendizagem escolar pode prestar uma contribuiçáo importante, colaborando no desenvolvimento de instrumentos diagnósticos e na elucidação dos mecanismos cognitivos envolvidos, bem como auxiliando a planejar estratégias mais eficientes de intervençáo, fundamentadas no conhecimento dos mecanismos cognitivos perturbados.

As dificuldades de aprendizagem da matemática (DAM) consistem em uma condição que altera a aquisição das habilidades de aritméticas. É um déficit de aprendizagem no qual a criança tem dificuldades persistentes na matemática (Butterworth, 2005). Indivíduos com DAM podem ter dificuldade em compreender conceitos numéricos simples, bem como em ter uma compreensão intuitiva de número. Além 
disso, podem ter problemas aprender fatos aritméticos e procedimentos matemáticos em geral. Ainda que eles produzam uma resposta correta ou usem o método correto, podem fazê-lo mecanicamente e sem confiança (Butterworth, 2005; Geary, 2005). As DAM são conhecidas por uma grande heterogeneidade, podendo estar relacionadas a mecanismos cognitivos diversos, tais como funçôes executivas e linguagem. Assim, o diagnóstico e o planejamento de intervençóes podem ser obscurecidos por outros déficits adjacentes, tornando-se um desafio ao neuropsicólogo.

O objetivo deste trabalho foi apresentar os mecanismos mais comumente apontados na literatura como subjacentes às DAM, através de uma revisão narrativa da literatura, baseada na escolha arbitrária de tópicos relevantes para a área. A partir dessa revisão, neuropsicólogos poderão conhecer melhor sobre os critérios para diagnóstico das DAM e auxiliar no tratamento, o que pode promover o desenvolvimento do capital mental de uma comunidade.

\section{Etiologia do Desempenho em Matemática e Mecanismos Neuropsicológicos}

Duas razóes podem ser identificadas como um foco central de pesquisa em relação aos mecanismos neurocognitivos subjacentes às DAM: a continuidade entre os níveis de desempenho e a heterogeneidade de mecanismos e manifestações (Haase, Moura, PinheiroChagas \& Wood, 2011). A natureza estatisticamente arbitrária do diagnóstico das DAM é corroborada por estudos geneticamente informados com pares de gêmeos, os quais indicam, por exemplo, que a etiologia do desempenho populacional em aritmética é multifatorial, sendo contínua a distribuição entre os indivíduos que apresentam maior ou menor desempenho (Wilccutt et al., 2010). O desempenho em aritmética é o resultado da interação de fatores genéticos e experienciais, e não há diferença qualitativa entre os níveis superior e inferior de desempenho.

O diagnóstico e a utilizaçáo de categorias nosológicas para se referir à aprendizagem da aritmética somente se justificam pelo fato de que as dificuldades nesta área são persistentes (Shalev, Manor \& Gross-Tsur, 2005), sendo associados a desfechos desfavoráveis não apenas do ponto de vista econômico (Bynner \& Parsons, 2006; Parsons \& Bynner, 1997), mas também psicossocial, como transtornos emocionais e comportamentais (Auerbach, Gross-Tsur, Manor \& Shalev, 2008) e até mesmo envolvimento com transgressóes legais (Bynner \& Parsons, 2006). Considerando a continuidade e a arbitrariedade dos critérios diagnósticos, bem como a necessidade de tornar os resultados de diferentes estudos mais comparáveis, Mazzocco (2007) fez uma proposta terminológica, a qual tem encontrado aceitaçáo na comunidade de pesquisadores. A recomendação de Mazzocco (2007) é a de que as crianças identificadas por um critério estatístico mais liberal de desempenho, por exemplo, aquelas cujo resultado em testes padronizados situase abaixo do percentil 25 sejam identificadas como portadoras de dificuldades de aprendizagem da matemática - DAM ou mathematical difficulties. Os dados de pesquisa mostram que, no grupo DAM, o rendimento escolar em matemática de um ano para outro é menos estável e a etiologia é mais variada, sendo maior a probabilidade de contribuição de variáveis socioemocionais e econômico-culturais (Mazzocco \& Myers, 2003; Mazzocco \& Thompson, 2005). Mazzocco (2007) sugeriu, por outro lado, que as crianças identificadas por um critério mais estrito de desempenho, como o percentil 5, fossem denominadas como portadoras de discalculia do desenvolvimento ou transtorno de aprendizagem da matemática - MLD ou mathematical learning disability. No grupo MLD, as dificuldades tendem a ser mais graves, persistentes e de etiologia constitucional. Apesar de reconhecermos a importância da terminologia sugerida, nesse texto será utilizado o termo mais genérico, dificuldades de aprendizagem da aritmética ou matemática (DAM), sem fazer uma distinção mais precisa em relação ao transtorno $\mathrm{da}$ aprendizagem da matemática (embora, ao se citar estudos, a terminologia usada pelos autores será respeitada). Julgamos que esta prática é justificável, na medida em que não parece, no momento, haver diferenças qualitativas entre os diversos grupos, e também pelo fato de a literatura disponível não ser suficiente para permitir um exame diferenciado da influência de diversos fatores sobre os três grupos: desenvolvimento típico, DAM e MLD.

O segundo motivo pelo qual o esclarecimento dos mecanismos das DAM adquire proeminência diz respeito à heterogeneidade clínica e etiológica. Além da etiologia multifatorial mencionada anteriormente (Willcutt et al., 2010), uma causa importante de dificuldades de aprendizagem da matemática diz respeito a síndromes de etiologia ambiental e genética. Algumas síndromes têm dificuldades com aritmética na sua expressão 
fenotípica, tais como embriopatia alcoólica (KoperaFrye, Dehaene \& Streissguth, 1996), síndrome de Turner (Bruandet, Molko, Cohen \& Dehaene, 2004), síndrome velocardiofacial (De Smedt et al., 2007) e hidrocefalia congênita precocemente tratada (Barnes, et al., 2006), etc. A associação com síndromes genéticas causadas por microdeleçóes indica que, além da etiologia multifatorial, as dificuldades de aprendizagem da matemática se devem a perturbações de loci gênicos específicos.

Além da heterogeneidade etiológica, as DAM se caracterizam também pela diversidade de manifestaçôes fenotípicas. A comorbidade entre dificuldades de aprendizagem de aritmética e outros transtornos é mais a regra do que a exceção (Rubinstein \& Henik, 2009). A comorbidade é alta, principalmente, com transtorno de aprendizagem da leitura (dislexia do desenvolvimento) e transtorno do déficit de atenção por hiperatividade (TDAH). No estudo de Gross-Tsur, Manor \& Shalev (1996), com uma coorte de 3.029 estudantes de 11 anos de idade, a prevalência de discalculia do desenvolvimento foi estimada em $6,5 \%$, enquanto as taxas de comorbidade para TDAH e para dislexia foram de 26 e 17\%, respectivamente. Em outra pesquisa (Dirks, Spyer, van Lieshout \& De Sonneville, 2008), em uma amostra de 799 crianças em idade escolar, 15\% daquelas diagnosticadas com transtornos de aprendizagem da matemática apresentavam transtornos da leitura e 7\% das crianças com transtornos de leitura apresentavam transtornos de aritmética.

A comorbidade com a dislexia do desenvolvimento poderia ser explicada por dificuldades no processamento fonológico, tais como a velocidade de nomeação rápida, a memória fonológica de curtoprazo e a consciência fonêmica (Jordan, 2007). A comorbidade com o TDAH, por outro lado, poderia encontrar sua explicação em comprometimentos da memória de trabalho e das funçôes executivas (Raghubar, Barnes \& Hecht, 2010). Entretanto, evidências de ocorrência independente e de mecanismos cognitivos distintos na discalculia e na dislexia do desenvolvimento foram obtidas em um estudo neurocognitivo de uma série de casos (Tressoldi, Rosati \& Lucangeli, 2007). No estudo de Tressoldi et al. (2007), foi observada uma dupla dissociação entre habilidades relacionadas ao senso numérico, comprometidas na discalculia, e habilidades relacionadas ao processamento fonológico, afetadas na dislexia. O mesmo tipo de dupla dissociação foi sugerido pelos resultados de um estudo com desenho transversal de grupo, conduzido por Landerl, Fussenegger, Moll e Willburger (2009). Sabe-se também que, apesar de o TDAH ser um fator de risco para dificuldades de aprendizagem, o diagnóstico é independente do nível intelectual e do desempenho escolar (Antshel et al., 2009).

Uma possível solução para o enigma das múltiplas associações entre agentes etiológicos e manifestaçôes fenotípicas de transtornos do desenvolvimento e da aprendizagem foi proposta a partir do conceito de endofenótipos (Bishop \& Rutter, 2009).

Endofenótipos são mediadores das relaçóes entre múltiplos mecanismos etiológicos (genéticos e ambientais) e múltiplas manifestações fenotípicas, uma vez que as relaçôes entre o genótipo e o fenótipo não são simples, sendo reguladas pelos tipos e níveis de atividade cerebral e, portanto, pela experiência do indivíduo (Waldman, 2005). Os endofenótipos são fenótipos intermediários, subjacente a transtornos que podem facilitar a detecção de riscos genéticos relativos a condição clínica complexa em questão (Archer, Oscar-Berman \& Blum, 2011). Por exemplo, memória de trabalho pode ser um endofenótipo em relação ao TDAH. Cada transtorno do desenvolvimento ou de aprendizagem pode, segundo esta perspectiva, ser caracterizado como um mosaico de endofenótipos. As manifestaçôes clínicas dependem de quais endofenótipos estão presentes em um determinado caso. Atualmente, os endofenótipos podem ser caracterizados através de métodos neuroquímicos, por neuroimagem funcional ou por meio de métodos neuropsicológicos.

Os principais endofenótipos neuropsicológicos implicados nas DAM dizem respeito a déficits no processamento fonológico, na memória de trabalho, nas habilidades visoespaciais e no senso numérico (Geary, 1993; Wilson \& Dehaene, 2007), os quais serão discutidos a seguir.

\section{Processamento Fonológico como Endofenótipo das Dificuldades de Aprendizagem da Matemática}

O processamento fonológico tem despertado interesse crescente em relação às DAM principalmente em função de sua comorbidade com as dificuldades de leitura (Simmons \& Singleton, 2007). Um estudo longitudinal conduzido por Hecht, Torgesen, Wagner e Rashotte (2001), composto por uma amostra de 201 crianças (médias de idades inicial e final de 7 e 
11 anos, respectivamente), mostrou que as habilidades de processamento fonológico avaliadas no $2^{\circ}$ ano eram preditivas do desempenho escolar na matemática até o $5^{\circ}$ ano. Hecht et al. (2001) consideram que o processamento fonológico é constituído de três subcomponentes. O primeiro dos componentes referese à velocidade de resgate das formas fonológicas da memória de longo-prazo, avaliada, geralmente, por meio de tarefas de nomeação automatizada rápida (tarefa em que o probando deve ver diversas figuras e nomeá-las o mais rápido possível). O segundo componente, por sua vez, diz respeito à capacidade de armazenamento na memória fonológica de curto-prazo, avaliada por meio de tarefas de alcance de apreensão (tarefas que avaliam quantos itens apresentados verbalmente um indivíduo consegue armazenar em sua memória de trabalho). E a consciência fonêmica, terceiro componente, o qual envolve o conhecimento da relaçáo grafema-fonema, usualmente avaliada por meio de tarefas de supressáo de fonemas (tarefa em que o indivíduo deve subtrair um fonema de uma palavra apresentada, e dizer como fica a nova palavra. Por exemplo, "pato" sem o [p] que se transforma e "ato"). Os resultados deste estudo (Hecht et al., 2001) mostraram que os três componentes do processamento fonológico constituem variância única para a predição do desempenho no $5^{\circ}$ ano a partir de avaliaçôes realizadas no $2^{\circ}$ ano. Apenas a consciência fonêmica foi preditiva do desempenho matemático a partir do $3^{\circ}$ e do $4^{\circ}$ ano. Hecht et al. (2001) interpretaram seus resultados considerando que a tarefa de supressão de fonemas exige habilidades cognitivas complexas, as quais se sobrepóem com o construto memória de trabalho.

$\mathrm{O}$ argumento quanto à importância do processamento fonológico para o desempenho em aritmética é, entretanto, enfraquecido por resultados de estudos que demonstram que os efeitos são eliminados quando se controla estatisticamente o efeito de variância relacionada à memória de trabalho (Berg, 2008; Berg \& Hutchinson, 2010; Swanson, 2004; Swanson \& Sachse-Lee, 2001). Assim, concluíse que o processamento fonológico desempenha um papel na aprendizagem dos aspectos da matemática que dependem mais fortemente do emprego de códigos verbais, apesar de seu papel possa ser sobreposto pelo processamento executivo e pela memória de trabalho. Cabe ressaltar que a influência da memória de trabalho na relação entre o processamento fonológico e o desempenho na matemática pode variar em função dos instrumentos de medida utilizados pelo estudo.

\section{Habilidade de Memória de Trabalho Subjacente ao Desempenho Matemático}

A importância da memória de trabalho para aprendizagem da aritmética está bem estabelecida, tanto no que se refere ao desenvolvimento típico quanto às dificuldades (Raghubar et al., 2010). O modelo teórico mais amplamente empregado é o componencial, original de Baddeley (2001). O modelo componencial pressupóe que a memória de trabalho é constituída por sistemas escravos representados por uma alça fonológica, por um bloco de notas visoespacial e por um buffer episódico, e por um executivo central (vide Raghubar et al., 2010 para uma revisão). $\mathrm{O}$ executivo central é especialmente importante por sustentar informaçóes durante um curto período de tempo, integrar informaçóes dos sistemas escravos, bem como se comunicar com a memória de longo prazo.

Um dos paradigmas experimentais mais frequentemente utilizados consiste em empregar tarefas de duplo processo e verificar quais componentes da memória de trabalho são mais prejudicados por tarefas interferentes. McKenzie, Bull \& Gray (2003) observaram, por exemplo, que o desempenho de crianças de 6 e 7 anos em operaçóes aritméticas era mais perturbado por tarefas interferentes de natureza visoespacial, enquanto o efeito sobre o desempenho de crianças mais velhas, com 8 e 9 anos, era mais perturbado por interferências de natureza verbal.

As tarefas de transcodificação entre as notaçôes verbal e arábica e vice-versa também demandam recursos de memória de trabalho. Em uma tarefa de ditado de numerais arábicos, Camos (2008) constatou que crianças com maior alcance de memória de trabalho em uma tarefa de alcance de quantidades contadas (counting span; tarefa em que o probando deve contar certas quantidades apresentadas em sequencia e, logo após, informar quais quantidades foram contadas) apresentavam desempenho superior àquelas com menor alcance de memória de trabalho, sendo o desempenho também correlacionado à complexidade dos itens a serem transcodificados. A complexidade dos itens, por sua vez, reflete o número de regras de transcodificação que se faziam necessárias empregar e, portanto, a carga de memória de trabalho demandada.

Um papel específico para a memória de trabalho visoespacial pôde ser inferido a partir de um estudo realizado por Zuber, Pixner, Moeller \& 
Nuerk (2009) com 130 crianças de 7 anos de idade, falantes nativas de alemão. Como se sabe, a notação numérica verbal na língua alemá se caracteriza pela inversão entre unidades e dezenas em números de dois algarismos. O número $78 \mathrm{em}$ alemão, por exemplo, é lido como acht (8) und siebzig (70) (oito e setenta). A inversão nos números com dois algarismos dificulta sobremaneira a aprendizagem dos números pelas crianças falantes de alemão. Zuber et al. (2009) descobriram que mais de 50\% dos erros cometidos pelas crianças eram de natureza sintática (por exemplo, escrever "79" em vez de "97"), envolvendo a inversão entre unidades e dezenas. Um dos principais preditores do desempenho neste tipo de erro foi a memória de trabalho visuoespacial, avaliada pela tarefa dos cubos de Corsi (tarefa em que o examinador toca alguns cubos, em uma sequência específica, e o probando deve repetir os movimentos do examinador na mesma ordem ou na ordem inversa. Para mais detalhes ver Kessels, van Zandvoort, Postma, Kappelle \& de Haan (2000).

A natureza da relação entre o executivo central e o desempenho em aritmética foi investigada em um trabalho conduzido por Bull \& Scerif (2001). As autoras investigaram as habilidades relacionadas à memória de trabalho em 93 crianças (média de idade $=7$ anos e 4 meses) com desenvolvimento típico ou com DAM. A avaliação baseou-se no modelo empiricamente derivado do executivo central, formulado por Miyake et al. (2000). Bull \& Scerif (2001) observaram comprometimentos de três componentes executivos da memória de trabalho em crianças com dificuldades de aprendizagem da aritmética, a saber: monitorização/fluência — Teste Wisconsin de Classificaçáo de Cartas (o probando deve agrupar cartas segundo um critério, o qual é inicialmente desconhecido, exigindo do respondente uma monitorização de seu comportamento para escolher o melhor critério) - updating - isto é, localizar o foco da memória de trabalho em novos estímulos - (Counting Span) e inibição de respostas prepotentes - Teste de Stroop (tarefa em que o probando deve inibir um estímulo interferente para execução de uma tarefa, como nomear a cor das letras em que o nome de outra cor é escrito).

A existência de déficits no executivo central em crianças com dificuldades de aprendizagem da matemática foi confirmada em um estudo muito engenhoso conduzido por van der Sluis, de Jong \& van der Leij (2004), utilizando um paradigma de subtração em uma amostra de 74 crianças da $4^{\mathrm{a}}$ e $5^{\mathrm{a}}$ séries. Os autores avaliaram as funçôes executivas, as quais se constituem em monitoramento e associaçáo de processos cognitivos envolvidos na execução de determinado comportamento. As funçóes executivas envolvem diversos subcomponentes, tais como controle inibitório, atenção, planejamento e tomada de decisão, e têm sido relacionadas a atividades do lobo frontal (Lezak, Howieson, Bigler, \& Tranel, 2012). O principal desafio de van der Sluis et al., (2004) era superar as impurezas típicas dos testes utilizados nas medidas de funçóes executivas. Para isso, foram desenhadas tarefas muito simples em diversas versôes, usando letras, algarismos ou figuras geométricas como estímulos. Na tarefa de nomeação, era simplesmente cronometrado o tempo que as crianças levavam para nomear algarismos arábicos apresentados em uma folha de papel. Na tarefa de quantificação, as crianças precisavam contar o número de elementos em conjuntos de estímulos, compostos por algarismos (por exemplo, 22 - "dois", 333 "três", 4444 - "quatro" etc.). As tarefas de nomeação e quantificação constituíam uma espécie de linha de base, uma vez que podiam ser resolvidas de forma rápida e automatizada.

A medida dependente no estudo de Van Der Sluis et al. (2004) foi o tempo adicional que as crianças levavam para executar a tarefa nas duas condiçóes experimentais - 1) indicar a quantidade de dígitos em um número, em condições incongruentes, por exemplo, para o número "222" a quantidade certa de dígitos é “3”; 2) ligar números e letras alternadamente, por exemplo 1-A-2-B... - comparativamente às duas condiçôes da linha de base. Os resultados mostraram que o custo temporal relacionado ao processamento controlado era significativamente maior nas crianças com dificuldades de aprendizagem da matemática do que no grupo com desenvolvimento típico. Os dados de pesquisa disponíveis contribuem então para formar a impressão de que três componentes do modelo componencial de memória de trabalho estáo envolvidos nas DAM.

\section{Habilidades Visoespaciais: Influência nas Dificuldades de Aprendizagem da Matemática}

A literatura neuropsicológica sobre acalculias adquiridas contribuiu para estabelecer solidamente a importância das habilidades de processamento visoespacial, principalmente no que se refere ao cálculo 
multidigital mental ou escrito (Hartje, 1987). Os erros procedimentais em operaçôes multidigitais de cálculo por escrito, tanto em crianças quanto em pacientes com acalculia, frequentemente envolvem erros que podem ser interpretados em função de disfunçôes do processamento visoespacial, tais como dificuldades com as operaçóes de troca e empréstimo entre uma coluna e outra, alinhamento das colunas (principalmente dos resultados intermediários) etc. Granà, Hofer \& Semenza (2006) descreveram um paciente com lesão do hemisfério esquerdo que apresentava um viés de procedimento, iniciando sempre todas as operaçóes de cálculo multidigital da esquerda para a direita, o que preserva as operaçôes de divisão, embora prejudique todas as demais. Alteraçóes graves no processamento numérico são, também, frequentemente observadas em pacientes com heminegligência visoespacial (Umiltà, Priftis \& Zorzi, 2009), as quais prejudicam as operaçôes de cálculo.

Tem sido mais difícil, entretanto, demonstrar um papel para os déficits visoespaciais nas DAM relacionadas a transtornos do desenvolvimento (Geary, 1993). Rourke (1989) postulou a existência de uma entidade denominada transtorno não verbal de aprendizagem (TNVA), a qual apresenta uma frequência estimada de $1 \%$ na populaçáo, comprometendo em graus variados a coordenação motora e a percepção somatossensorial, as habilidades visoespaciais, o desempenho em aritmética, a capacidade inferencial não verbal e as habilidades sociocognitivas (vide revisões em Davis \& Broitman, 2011; Volden, 2004). Venneri, Cornoldi \& Garuti (2003) observaram que crianças com um quadro neuropsicológico compatível com TNVA apresentavam maiores dificuldades em cálculo multidigital envolvendo operaçôes de transferência entre colunas, o que é sugestivo de uma dificuldade no processamento visoespacial. As razóes para o fracasso em identificar de modo mais claro o papel dos mecanismos de processamento visoespacial nos transtornos de aprendizagem da matemática podem estar relacionadas, em parte, à baixa prevalência do TNVA. A maioria dos estudos emprega a estratégia de recrutar crianças da população escolar e, posteriormente, estratificá-las conforme o nível de rendimento em matemática (Fuchs et al., 2010; Landerl et al., 2009; Landerl \& Moll, 2010). Esta estratégia de recrutamento na populaçáo em geral é claramente fadada ao fracasso se considerarmos que o TNVA tem uma frequência populacional estimada em torno de 1\%. Há necessidade de realizar pesquisas com amostras clínicas. A outra razão pode estar relacionada, também, ao fato de que raros estudos, como o de Venneri et al. (2003), investigam habilidades mais complexas, como o cálculo multidigital em crianças com dificuldades de aprendizagem. A literatura neuropsicológica com adultos sugere, entretanto, que são as operaçóes de cálculo mais complexas, justamente, aquelas mais afetadas pelos déficits visoespaciais.

\section{Senso Numérico: Mecanismo Específico das Dificuldades de Aprendizagem da Matemática}

A análise das dificuldades de aprendizagem da aritmética em termos de mecanismos cognitivos específicos é, geralmente, realizada no âmbito do modelo de triplo código (Dehaene, 1992; Dehaene \& Cohen, 1995, vide também revisão em Haase, Wood \& Willmes, 2010). Segundo o modelo de código triplo, as representaçôes não simbólicas são relacionadas à atividade de áreas do sulco intraparietal bilateralmente, as quais são ativadas toda vez que o conteúdo semântico de magnitude precisa ser acessado. A automatização dos fatos aritméticos se dá através da consolidação de representaçôes verbais, cujo acesso depende do giro angular bilateralmente. Finalmente, a utilizaçáo dos algoritmos de cálculo no sistema arábico é possível a partir da ativação destas representações no giro fusiforme, bilateralmente (Dehaene, Piazza, Pinel \& Cohen, 2003). Em termos de mecanismos cognitivos específicos, a aprendizagem da matemática pode ser explicada por este modelo.

O modelo de triplo código considera que as representaçôes verbais (três) e arábicas (3) de numerosidade são construçôes culturais feitas a partir de uma forma mais primitiva de representação não simbólica de magnitude $(\diamond \bullet)$, a qual é compartilhada com diversos animais (Piazza \& Dehaene, 2004). As representaçóes não simbólicas podem ser testadas, por exemplo, em tarefas de comparação da magnitude de conjuntos de pontos, sendo caracterizada pela lei psicofísica de WeberFechner. O efeito da distância corresponde à lei de Weber: quanto menor a distância numérica entre os conjuntos a serem discriminados, maior é o tempo de reaçáo e a taxa de erros. A lei de Fechner corresponde ao fato de que a função psicofísica que melhor se ajusta aos tempos de reação do probando é logaritmicamente comprimida. Isto é, quanto maior 
a magnitude considerada, menor é a precisão da sua representação (Izard \& Dehaene, 2008).

Os primeiros estudos que procuraram caracterizar um déficit no senso numérico em crianças com dificuldades de aprendizagem da aritmética utilizaram-se do paradigma de comparação de magnitudes (Landerl, Bevan \& Butterworth, 2004; Rousselle \& Noël, 2007). Na versão simbólica, a tarefa de comparação de magnitudes envolve discriminar se um determinado numeral arábico apresentado na tela do computador é maior ou menor do que um determinado valor de referência. $\mathrm{Na}$ versão não simbólica da tarefa, são apresentados dois conjuntos de pontos simultaneamente, e o examinado deve decidir qual é o maior. Além de medidas do tempo de reação e da acurácia, procurase observar com este paradigma o chamado efeito da distância. Por exemplo, a taxa de erros e o tempo de reação são inversamente correlacionados à distância numérica entre os estímulos, indicando que o sistema de discriminação de magnitudes apresenta um limite de resolução ou acurácia, o qual corresponde às previsóes feitas pela lei de Weber.

Maiores tempos de reação e taxas de erro na tarefa de comparação simbólica de magnitudes em crianças com DAM foram observados em diversos estudos (Bachot, Gevers, Fias \& Roeyers, 2005; De Smedt \& Gilmore, 2011; De Smedt et al., 2007; Landerl et al., 2004; Rousselle \& Noël, 2007). Não foi possível, entretanto, demonstrar de forma consistente diferenças entre os dois grupos em tarefas de comparação não simbólica de magnitude (De Smedt \& Gilmore, 2011; Rousselle \& Noël, 2007). Uma interpretaçáo proposta para este resultado consiste em postular que a dificuldade das crianças não se encontra tanto no senso numérico em si, mas sim, no acesso automático às representações não simbólicas de magnitude a partir das representaçóes simbólicas. Não se trata, portanto, de um déficit representacional de magnitude, mas de um déficit de acesso. A hipótese de acesso foi corroborada por Rubinstein e Henik (2005), usando um paradigma de tipo Stroop em uma amostra de 38 indivíduos, sendo metade deles diagnosticados com discalculia (média de idade $=24$ anos e 2 meses) e a outra metade constituinte do grupo controle (média de idade $=23$ anos e 9 meses). $\mathrm{Na}$ tarefa de interferência número-tamanho são apresentados pares de algarismos numéricos, e o examinando precisa decidir qual é o número maior em magnitude (por exemplo, "5" x "8") ou em tamanho físico (fonte maior, por exemplo " 5 " $\mathrm{x}$ "8"). Os estímulos são apresentados de forma que as relaçóes entre o tamanho e o número podem ser congruentes (maior número, maior tamanho), incongruentes (menor número, maior tamanho ou maior número, menor tamanho) e neutros (os dois estímulos do mesmo tamanho).

Um passo importante no sentido de demonstrar a relevância do senso numérico para a aprendizagem da matemática foi dado por Halberda, Mazzocco \& Feigenson (2008). Trabalhando com 64 jovens (média de idade=14 anos) da população em geral, Halberda et al. (2008) demonstraram que a acuidade numérica, ou seja, a fração de Weber (w) para discriminação de magnitudes não simbólicas, apresentava distribuição normal e se correlacionava negativamente com o desempenho em aritmética (isto é, melhor desempenho na tarefa de comparação, melhor desempenho na aritmética). Posteriormente, Piazza et al. (2010) demonstraram que crianças com DAM apresentam uma menor acuidade numérica na tarefa de comparação não simbólica de magnitudes. Este resultado foi confirmado por Ferreira et al. (2012), Costa et al. (2011) e Mazzocco, Feigenson \& Halberda (2011). No estudo de Piazza et al. (2010), a fração de Weber estimada para crianças de 10 anos $(\mathrm{n}=23)$ com DAM foi de $\mathrm{w}=0,34$, sendo equivalente àquela apresentada por crianças de 5 anos $(n=26)$ com desenvolvimento típico. Crianças de 10 anos com desenvolvimento típico $(n=26)$ apresentam uma fração de Weber da ordem de $\mathrm{w}=0,25$, sendo o valor médio para adultos $(\mathrm{n}=20)$ igual a $\mathrm{w}=0,15$.

Os resultados dos estudos mais recentes confirmam, portanto, que a acuidade numérica não simbólica — ou senso numérico — pode estar comprometida em indivíduos com DAM. Não se conhece, entretanto, a frequência com que este déficit ocorre, o seu poder preditivo em relação a outras variáveis e a fase do desenvolvimento na qual sua influência é determinante. Ainda são poucos os estudos integrativos que analisam, de forma multivariada, o poder preditivo das diversas fontes de influência.

\section{Modelos Integrativos das Dificuldades de Aprendizagem da Matemática}

A revisão realizada mostrou que a aprendizagem da aritmética, tanto em crianças com desenvolvimento típico quanto em crianças com DAM, é influenciada por diversos fatores genéticos e experienciais. Os principais 
fatores cognitivos podem ser inespecíficos à matemática, tais como o processamento fonológico, a memória de trabalho ou as habilidades de processamento visoespacial. Mas fatores inerentes à cognição matemática, também são importantes, uma vez que influenciam o desempenho em aritmética. Ainda não está esclarecido o poder preditivo relativo de cada um destes fatores. Para isto, faltam mais estudos integrativos, usando técnicas de análise multivariada. A maioria dos estudos não examina simultaneamente o efeito de todas as variáveis potencialmente relevantes. Todavia, alguns passos já foram dados nesta direção, como os estudos conduzidos por Fuchs et al. (2010) e por LeFevre et al. (2010).

No estudo de Fuchs et al. (2010), foi investigada uma amostra representativa de mais de 200 crianças do $1^{\circ}$ ano do ensino fundamental. As medidas preditoras abrangiam uma ampla gama de fatores cognitivos gerais (vocabulário, compreensão de sentenças, memória de trabalho, além de uma escala semiquantitativa para avaliaçáo de comportamentos desatentos). Os fatores específicos, por outro lado, eram representados pela tarefa da linha numérica (era apresentado um desenho de termômetro ao examinando, o qual deveria indicar onde se localizavam temperaturas-alvo) e pelo Number Sets Test (Geary, Bailey, \& Hoard, 2009). Diversos modelos de regressão múltipla mostraram que tanto fatores gerais quanto inespecíficos influenciavam os dois principais desfechos analisados, a capacidade de computação aritmética e a resolução de problemas verbalmente formulados. Os preditores de natureza específica se associaram às duas medidas de desfecho. Por outro lado, o comportamento desatento associouse negativamente com as operaçôes aritméticas, enquanto a memória de trabalho e as tarefas verbais se correlacionaram com a solução de problemas verbalmente formulados. $\mathrm{O}$ estudo de Fuchs et al. (2010) pode ser criticado, entretanto, porque as medidas preditoras não podem ser consideradas como medidas genuínas do senso numérico. Tanto a tarefa do termômetro quanto o Number Sets Test misturam representaçôes simbólicas e não simbólicas de numerosidade. Adicionalmente, o Number Sets Test é uma tarefa cronometrada, na qual a criança precisa tanto comparar quanto realizar operaçôes sobre pequenos conjuntos de objetos ou de números, já que o resultado da criança pode ter sido obscurecido pela sua velocidade de processamento geral. Ainda assim, o estudo contribuiu mostrando a importância tanto de fatores cognitivos gerais quanto específicos para a matemática.
O estudo conduzido por Lefevre et al. (2010), cujo tamanho da amostra era de 182 crianças com idade variada entre 4,5 e 7,5 anos, aproxima-se melhor dos requisitos necessários para se examinar a importância relativa dos diversos tipos de fatores cognitivos. O estudo foi longitudinal, iniciando-se no jardim da infância e acompanhando-se as crianças por três anos. Foi testado um modelo de equação estrutural, composto por três vias principais, as quais se mostraram significativas $\mathrm{e}$ independentes. $\mathrm{Na}$ via do senso numérico, o tempo de latência para estimar a grandeza de conjuntos de pontos com até seis elementos foi preditivo da capacidade de operaçôes aritméticas não verbais. $\mathrm{Na}$ via linguística, o vocabulário receptivo e a tarefa de supressáo de fonemas foram preditores da capacidade de transcodificação numérica. Finalmente, na via espacial, foi observada uma associaçáo entre o desempenho em uma tarefa semelhante aos cubos de Corsi e os dois tipos de desfecho.

Pode-se criticar o estudo de LeFevre et al. (2010) porque a medida preditora do senso numérico situa-se na faixa de conjuntos até seis elementos. A habilidade de estimar precisa e rapidamente a grandeza de conjuntos de até quatro ou cinco elementos é conhecida como subitizing e representa um domínio distinto do senso numérico aproximativo e não simbólico descrito no modelo de código triplo (Feigenson, Dehaene \& Spelke, 2004). A associação encontrada entre as habilidades de subitizing e o desempenho ulterior em aritmética não verbal é interessante e merece investigação mais detalhada. Entretanto, há necessidade de se realizar um estudo que compare o poder preditivo do senso numérico ou da acuidade numérica, operacionalizada como a fração de Weber em uma tarefa de comparação não simbólica da magnitude de conjuntos, em contraste a outros fatores cognitivos, tais como o processamento visoespacial, fonológico e os diversos componentes da memória de trabalho.

\section{Conclusão}

Tendo em vista os estudos apresentados nesta revisão, observa-se uma heterogeneidade presente nas DAM, as quais são influenciadas por múltiplos fatores epigenéticos, como memória de trabalho, processamento fonológico, habilidades visoespaciais e senso numérico. Além disso, existe uma variedade de endofenótipos que podem afetar em diferentes graus o desempenho aritmético, caracterizando perfis distintos de dificuldade de aprendizagem da matemática. 
Além disso, diversas questôes adicionais permanecem sem resposta. Por exemplo, as diferenças individuais quanto aos fatores cognitivos cruciais à aprendizagem da aritmética, a especificidade dos preditores em relação aos múltiplos desfechos representados pela complexidade e natureza hierárquica das habilidades aritméticas e, finalmente, ao efeito de diversos fatores cognitivos, os quais têm destaques diferenciais conforme a fase do desenvolvimento da criança. A memória de trabalho visoespacial pode ser mais importante no início da aprendizagem da aritmética, crescendo a importância dos fatores fonológicos à medida que a criança avança no currículo. Da mesma forma, o senso numérico pode ser mais importante no início da aprendizagem. Pode ocorrer, também, que as crianças com DAM não apresentem um déficit absoluto, mas, sim, uma maturação mais lenta das habilidades relacionadas ao senso numérico.

Assim, apesar da falta de estudos mais robustos, principalmente, sobre o desenvolvimento específico dos endofenótipos de dificuldade de aprendizagem, o fato de se conhecer os mecanismos subjacentes à aprendizagem da matemática e os critérios de diagnóstico das DAM pode auxiliar na melhor caracterização clínica para essa população. Por consequência, a formulação de intervençôes mais específicas para indivíduos com aprendizagem deficitária da aritmética pode auxiliar no desenvolvimento do capital mental de uma comunidade.

\section{Referências}

Antshel, K. M., Faraone, S. V., Maglione, K., Doyle, A., Fried, R., Seidman, L., \& Biederman, J. (2009). Is adult attention deficit hyperactivity disorder a valid diagnosis in the presence of high IQ? Psychological Medicine, 39(8), 1325-1335.

Archer, T., Oscar-Berman, M., \& Blum, K. (2011). Epigenetics in developmental disorder: adhd and endophenotypes. Journal of Genetic Syndrome \& Gene Therapy, 2(104), 1000104.

Auerbach, J. G., Gross-Tsur, V., Manor, O., \& Shalev, R. S. (2008). Emotional and behavioral characteristics over a six-year period in youths with persistent and nonpersistent dyscalculia. Journal of Learning Disabilities, 41(3), 263-273.

Bachot, J., Gevers, W., Fias, W., \& Roeyers, H. (2005). Number sense in children with visuospatial disabilities:orientation of the mental number line. Psychology Science, 47, 172-183.

Baddeley, A. (2001). The magic number and the episodic buffer. Behavioral and Brain Sciences, 24(1), 117-118.

Barnes, M. A., Wilkinson, M., Khemani, E., Boudesquie, A., Dennis, M. \& Fletcher, J. M. (2006). Arithmetic processing in children with spina bifida: Calculation accuracy, strategy use, and fact retrieval fluency. Journal of Learning Disabilities, 39(2), 174-187.

Berg, D. H. (2008). Working memory and arithmetic calculation: the contributory role of processing speed, short-term meory and reading. Journal of Experimental Child Psychology, 99(4), 288-308.

Berg, D. H., \& Hutchinson, N. L. (2010). Cognitive processes that account for addition fluency differences between typically achieving in arithmetic and children at-risk for failure in arithmetic. Learning Disabilities: A Contemporary journal, 8, 1-20.

Bishop, D. V. M., \& Rutter, M. (2009). Neurodevelopmental disorders: conceptual issues. In M. Rutter, D. V. M. Bishop, D. S. Pine, S. Scott, J. Stevenson, E. Taylor \& A. Thapar (Orgs.), Rutter's child and adolescent psychiatry, (5a ed., pp. 32-41). Oxford: Blacwell.

Bruandet, M., Molko, N., Cohen, L., \& Dehaene, S. (2004). A cognitive characterization of dyscalculia in Turner syndrome. Neuropsychologia, 42(3), 288-298.

Bull, R., \& Scerif, G. (2001). Executive functioning as a predictor of children's mathematics ability: inhibition, switching, and working memory. Developmental Neuropsychology, 19(3), 273-293.

Butterworth, B. (2005). The development of arithmetical abilities. The Journal of Child Psychology and Psychiatry and Allied Disciplines, 46(1), 3-18.

Bynner, J., \& Parsons, S. (2006). Does Numeracy Matter More? Education. National Research and Development Centre for Adult Literacy and Numeracy, Institute of Education, University of London.

Camos, V. (2008). Low working memory capacity impedes both efficiency and learning of number transcoding in children. Journal of Experimental Child Psychology, 99(1), 37-57.

Cooper, C. L., Field, J., Goswami, U., Jenkins, R., \& Sahakian, B. J. (2010). Mental capital and wellbeing. Oxford: Wiley-Blackwell. 
Costa, A. J., Silva, J. B. L., Chagas, P. P., Krinzinger, H., Lonneman, J., Willmes, K., Wood, G., \& Haase, V. G. (2011). A hand full of numbers: a role for offloadingin arithmetics learning? Frontiers in Psychology, 2, 368.

Davis, J. M., \& Broitman, J. (2011). Nonverbal learning disabilities in children. Bridging the gap between science and practice. New York: Springer.

De Smedt, B., Swillen, A., Devriendt, K., Fryns, J. P., Verschaffel, L., \& Ghesquiere, P. (2007). Mathematical disabilities in children with velo-cardiofacial syndrome. Neuropsychologia, 45(5), 885-895.

De Smedt, B., \& Gilmore, C. K. (2011). Defective number module or impaired access? Numerical magnitude processing in first graders with mathematical difficulties. Journal of Experimental Child Psychology, 108(2), 278-292.

Dehaene, S. (1992). Varieties of numerical abilities. Cognition, 44(1-2), 1-42.

Dehaene, S., \& Cohen, L. (1995). Towards an anatomical and functional model of number processing. Mathematical Cognition, 1(1), 83-120.

Dehaene, S., Piazza, M., Pinel, P., \& Cohen, L. (2003). Three parietal circuits for number processing. Cognitive Neuropsychology, 20(3), 487-506.

Dirks, E., Spyer, G., van Lieshout, E. C. D. M., \& De Sonneville, L. (2008). Prevalence of combined reading and arithmetic disabilities. Journal of Learning Disabilities, 41(5), 460-473.

Feigenson, L., Dehaene, S., \& Spelke, E. (2004). Core systems of number. Trends in Cognitive Sciences, 8(7), 307-314.

Felder-Puig, R., Baumgartner, M., Topf, R., Gadner, H., \& Formann, A. K. (2008). Health-related quality of life in Austrian elementary school children. Medical Care, 46(4), 432-439.

Ferreira, F. O., Wood, G., Pinheiro-Chagas, P., Lonnemann, J., Krinzinger, H., Willmes, K., \& Haase, V. (2012). Explaining school mathematics performance from symbolic and nonsymbolic magnitude processing: similarities and differences between typical and low-achieving children. Psychology \& Neuroscience, 5(1) 37-46.

Fuchs, L. S., Geary, D. C., Compton, D. L., Fuchs, D., Hamlett, C. L., \& Bryant, J. D. (2010). The contributions of numerosity and domain-general abilities to school readiness. Child Development, 81(5), 1520-1533.

Geary, D. C. (1993). Mathematical disabilities: cognitive, neuropsychological, and genetic components. Psychological Bulletin, 114(2), 345-62.
Geary, D. C. (2005). Role of cognitive theory in the study of learning disability in mathematics. Journal of Learning Disabilities, 38(4), 305-307.

Geary, D. C., Bailey, D. H., \& Hoard, M. K. (2009). Predicting mathematical achievement and mathematical learning disability with a simple screening tool: The Number Sets Test. Journal of Psychoeducational Assessment, 27(3), 265-279.

Granà, A., Hofer, R., \& Semenza, C. (2006). Acalculia from a right hemisphere lesion dealing with "where" in multiplication procedures. Neuropsychologia, 44(14), 2972-2986.

Gross-Tsur, V., Manor, O., \& Shalev, R. S. (1996). Developmental dyscalculia: prevalence and demographic features. Developmental Medicine and Child Neurology, 38(1), 25-33.

Haase, V. G., Wood, G., \& Willmes, K. (2010). Matemática. In L. F. Malloy-Diniz, D. Fuentes, P. Mattos \& N. Abreu. (Eds.), Avaliação neuropsicológica, (pp. 123-132), Porto Alegre: ARTMED.

Haase, V. G., Moura, R. J., Pinheiro-Chagas, P., \& Wood, G. (2011). Discalculia e dislexia: semelhança epidemiológica e diversidade de mecanismos neurocognitivos. In L. M. Alves, R. Mousinho \& S. A. Capellini (Eds.), Dislexia: novos temas, novas perspectivas, (pp. 257-282), Rio de Janeiro: Wak.

Halberda, J., Mazzocco, M. M. M., \& Feigenson, L. (2008). Individual differences in non-verbal number acuity correlate with maths achievement. Nature, 455(7213), 665-668.

Hartje, W. (1987). The effect of spatial disorders on arithmetical skills. In G. Deloche \& X. Seron (Eds.), Mathematical disabilities, (pp. 121-125), Hillsdale, NJ: Erlbaum.

Hecht, S. A., Torgesen, J. K., Wagner, R. K., \& Rashotte, C. A. (2001). The relations between phonological processing abilities and emerging individual differences in mathematical computation skills: a longitudinal study from second to fifth grades. Journal of Experimental Child Psychology, 79(2), 192-227.

Izard, V., \& Dehaene, S. (2008). Calibrating the mental number line. Cognition, 106(3), 1221-1247.

Jordan, N. C. (2007). Do words count? Connections between mathematics and reading difficulties. In D. B. Berch \& M. M. M. Mazzocco (Eds.), Why is math so hard for some children? The nature and origins of mathematical learning difficulties and disabilities, (pp. 107-120), Baltimore, MA: Brookes. 
Kessels, R. P., van Zandvoort, M. J., Postma, A., Kappelle, L. J., \& de Haan, E. H. (2000). The Corsi Block-Tapping Task: standardization and normative data. Applied Neuropsychology, 7(4), 252-258.

Kopera-Frye K., Dehaene S., \& Streissguth A. P. (1996). Impairments of number processing induced by prenatal alcohol exposure. Neuropsychologia, 34(12), 1187-1196.

Landerl, K., Bevan, A., \& Butterworth, B. (2004). Developmental dyscalculia and basic numerical capacities: A study of 8-9-yearold students. Cognition, 93(2), 99-125.

Landerl, K., Fussenegger, V., Moll, K., \& Willburger, E. (2009). Dyslexia and dyscalculia: two learning disorders with different cognitive profiles. Journal of Experimental Child Psychology, 103(3), 309-324.

Landerl, K., \& Moll, K. (2010). Comorbidity of learning disorders: prevalence and familial transmission. The Journal of Child Psychology and Psychiatry, and Allied Disciplines, 51(3), 287-294.

LeFevre, J. A., Fast, L., Skwarchuk, S. L., SmithChant, B. L., Bisanz, J., Kamawar, D., \& PennerWilger, M. (2010). Pathways to mathematics: longitudinal predictors of performance. Child Development, 81(6), 1753-1767.

Lezak, M. D., Howieson, D. B., Bigler, D. B., \& Tranel, D. (2012). Neuropsychological Assessment (5th ed., pp. 666-711).

Mazzocco, M. M. M., \& Myers, G. F. (2003). Complexities in identifying and defining mathematics learning disability in the primary school-age years. Annals of Dyslexia, 53(1), 218-253.

Mazzocco, M. M. M., \& Thompson, R. E. (2005). Kindergarten predictors of math learning disability. Learning Disabilities Research \& Practice: a publication of the Division for Learning Disabilities, Council for Exceptional Children, 20(3), 142-155.

Mazzocco, M. M. M. (2007). Defining and differentiating mathematical learning disabilities and difficulties. In D. B. Berch \& M. M. M. Mazzocco (Eds.), Why is math so hard for some children? The nature and origins of mathematical learning difficulties and disabilities, (pp. 29-47), Baltimore: Brookes.

Mazzocco, M. M., Feigenson, L., \& Halberda, J. (2011). Impaired acuity of the approximate number system underlies mathematical learning disability (dyscalculia). Child Development, 82(4), 1224-1237.
McKenzie, B., Bull, R., \& Gray, C. (2003). The effects of phonological and visual-spatial interference on children's arithmetical performance. Educational and Child Psychology, 20(3), 93-108.

Miyake, A., Friedman, N. P., Emerson, M. J., Witzki, A. H., Howerter, A., \& Wager, T. D. (2000). The unity and diversity of executive functions and their contributions to complex "Frontal Lobe" tasks: a latent variable analysis. Cognitive Psychology, 41(1), 49-100.

Parsons, S., \& Bynner, J. (1997). Numeracy and employment. Education + Training, 39(2), 43-51.

Piazza, M., \& Dehaene, S. (2004). From number neurons to mental arithmetic: the cognitive neuroscience of number sense. In M. Gazzaniga, (Ed.), The Cognitive Neurosciences, (pp. 865-875), Cambridge: MIT Press Book.

Piazza, M., Facoetti, A., Trussardi, A. N., Berteletti, I., Conte, S., Lucangeli, D., Dehaene, S., \& Zorzi, M. (2010). Developmental trajectory of number acuity reveals a severe impairment in developmental dyscalculia. Cognition, 116(1), 33-41.

Raghubar, K. P., Barnes, M. A., \& Hecht, S. A. (2010). Working memory and mathematics: A review of developmental, individual difference, and cognitive approaches. Learning and Individual Differences, 20(2), 110-122.

Rourke, B. P. (1989). Nonverbal learning disability. The syndrome and the model. New York: Guilford.

Rousselle, L., \& Noël, M. P. (2007). Basic numerical skills in children with mathematics learning disabilities: a comparison of symbolic vs. nonsymbolic number magnitude processing. Cognition, 102(3), 361-395.

Rubinsten, O., \& Henik, A. (2005). Automatic activation of internal magnitudes: a study of developmental dyscalculia. Neuropsychology, 19(5), 641-648.

Rubinstein, O., \& Henik, A. (2009). Developmental dyscalculia: heterogeneity might not mean different mechanisms. Trends in Cognitive Sciences, 13(2), 92-99.

Shalev, R. S., Manor, O., \& Gross-Tsur, V. (2005). Developmental dyscalculia: a prospective six-year follow-up. Developmental Medicine and Child Neurology, 47(2), 121-125.

Simmons, F. R., \& Singleton, C. (2007). Do weak phonological representations impact on arithmetic development? A review of research into arithmetic and dyslexia. Dyslexia, 14(2), 77-94. 
Swanson, H. L., \& Sachse-Lee, C. (2001). Mathematical problem solving and working memory in children with learning disabilites: both executive and phonological processes are important. Journal of Experimental Child Psychology, 79(3), 294-321.

Swanson, H. L. (2004). Working memory and phonological processing as predictors of children's mathematical problem solving at different ages. Memory \& Cognition, 32(4), 648-661.

Tressoldi, P. E., Rosati, M., \& Lucangeli. D. (2007). Patterns of developmental dyscalculia with or without dyslexia. Neurocase, 13(4), 217-225.

Umiltà, C., Priftis, K., \& Zorzi, M. (2009). The spatial representation of numbers: evidence from neglect and pseudoneglect. Experimental Brain Research, 192(3), 561-569.

Van Der Sluis, S., de Jong, P. F., \& Van Der Leij, A. (2004). Inhibition and shifting in children with learning deficits in arithmetic and reading. Journal of Experimental Child Psychology, 87(3), 239-266.

Venneri, A., Cornoldi, C., \& Garuti, M. (2003). Arithmetic difficulties in children with visuospatial learning disability (VLD). Child neuropsychology: a journal on normal and abnormal development in childhood and adolescence, 9(3), 175-183.

Volden, J. (2004). Nonverbal learning disability: a tutorial for speech-language pathologists. American Journal of Speech-Language Pathology, 13(2), 128-141.

Waldman, I. D. (2005). Statistical approaches to complex phenotypes: evaluating neuropsychological endophenotypes for attentiondeficit/hyperactivity disorder. Biological Psychiatry, 57(11), 1347-1356.

Willcutt, E. G., Pennington, B. F., Duncan, L., Smith, S. D., Keenan, J. M., Wadsworth, S., Defries, J. C., \& Olson, R. K. (2010). Understanding the complex etiologies of developmental disorders: behavioral and molecular genetic approaches. Journal of Developmental \& Behavioral Pediatrics, 31(7), 533-544.

Wilson, A. J., \& Dehaene, S. (2007). Number sense and developmental dyscalculia. In D. Coch, K. Fischer \& G. Dawson (Eds), Human Behavior and the Developing Brain, New York: Guilford Press.

Zuber, J., Pixner, S., Moeller, K., \& Nuerk, H. C. (2009). On the language specificity of basic number processing: transcoding in a language with inversion and its relation to working memory capacity. Journal of Experimental Child Psychology, 102(1), 60-77.

\section{Endereço para correspondência:}

Vitor Geraldi Haase

Universidade Federal de Minas Gerais, Avenida Antônio Carlos, 6.627

CEP 31270-901 - Belo Horizonte/MG

E-mail:vghaase@gmail.com

Recebido em 05/08/2012

Revisto em 20/10/2012

Aceito em 05/11/2012 